International Journal of Pure and Applied Mathematics

Volume 98 No. 3 2015, 375-387

ISSN: 1311-8080 (printed version); ISSN: 1314-3395 (on-line version)

url: http://www.ijpam.eu

doi: http://dx.doi.org/10.12732/ijpam.v98i3.10

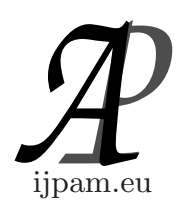

\title{
MAX-MIN INTUITIONISTIC FUZZY MATRIX OF AN INTUITIONISTIC FUZZY GRAPH
}

\author{
G. Deepa ${ }^{1 \S}$, B. Praba ${ }^{2}$, V.M. Chandrasekaran ${ }^{3}$ \\ ${ }^{1,3}$ School of Advanced Sciences \\ VIT University \\ Vellore, 632014, Tamilnadu, INDIA \\ ${ }^{2} \mathrm{SSN}$ College of Engineering \\ Kalavakkam, Chennai, 603110, Tamilnadu, INDIA
}

\begin{abstract}
In this paper we introduce the Max-Min intuitionistic fuzzy matrix $M(G)$ of an intuitionistic fuzzy graph. And the extreme energy of $M(G)$ is defined. And also we give the explicit expression for the coefficients of the characteristic polynomial of $M(G)$. These concepts are illustrated with real time example.
\end{abstract}

AMS Subject Classification: 03E72, 05C50

Key Words: intuitionistic fuzzy set, intuitionistic fuzzy graph, energy of fuzzy graph

\section{Introduction}

The foundation for graph theory was laid in 1735 by Leonhard Euler when he solved the 'Konigsberg bridges' problem. Many real life problems can be represented by graph. In computer science, graphs are used to represent net-

Received: October 20, 2014

(C) 2015 Academic Publications, Ltd.

$\S$ Correspondence author url: www.acadpubl.eu 
works of communications, data organization, computational devices, the flow of computation, etc. The link structure of a website could be represented by a directed graph in which the vertices are the web pages available at the website and a directed edge from page $\mathrm{A}$ to page $\mathrm{B}$ exists if and only if $\mathrm{A}$ contains a link to B (see [14]). A similar approach can be taken to problems in travel, biology, computer chip design and many other fields. Hence graph theory is widely used in solving real time problems. But when the system is large and complex it is difficult to extract the exact information about the system using the classical graph theory. In such cases fuzzy graph is used to analyze the system. In 1973, the definition of fuzzy graph was introduced by Kafmann (see [10]) from the Zadeh's fuzzy relations. In 1975, Rosenfeld (see [16]) introduced another detailed definition including fuzzy vertex and fuzzy edges and several fuzzy analogs of graph theoretic concepts such as paths, cycles, connectedness and etc. In 1999, the first definition of intuitionistic fuzzy graph was proposed by Atanassov (see [2]). In 1978, Gutman (see [8]) introduced the energy of a graph as the sum of the absolute values of the eigen values of the adjacency matrix of a graph. The lower and upper bound for the energy of a graph are discussed in (see [4], [12], [9]). The energy of fuzzy graph and its bounds are discussed in (see [1]). The energy of an intuitionistic fuzzy graph and its bounds are discussed in (see [15]). In (see [15]), the adjacency matrix of an intuitionistic fuzzy graph is defined. Using this adjacency matrix we defined the energy of an intuitionistic fuzzy graph and the lower and upper bounds for the energy of an intuitionistic fuzzy graph are obtained. These concepts are illustrated with real time example. The link structure of a website could be represented by an intuitionistic fuzzy directed graph. The links are considered as vertices and the path between the links are considered as edges. The weightage of the each edge are considered as the number of visitors (membership value), the number of non visitors (non membership value) and drop off (intuitionistic fuzzy index) among that link structure. In (see [7]), The authors discussed the virus spread in an intuitionistic fuzzy network. Each node (vertex) in this network is either infected or healthy. An infected node can infect its neighbours with an infection rate $\beta$, and it is cured with curing rate $\delta$. The ratio $\tau=\frac{\beta}{\delta}$ is called the effective spreading rate of the virus spread in an intuitionistic fuzzy network. Also the sharp epidemic threshold $\tau_{c}$ of the virus spread in an intuitionistic fuzzy network is defined. Many authors (see [17], [3], [6], [11], [13]) discussed the existence of an epidemic threshold $\tau_{c}$. If the effective spreading rate $\tau=\frac{\beta}{\delta}>\tau_{c}$, then virus continue and a nonzero fraction of the nodes are infected, whereas $\tau \leq \tau_{c}$, the epidemic dies out. The relationship between the number of visitors and the energy of an intuitionistic fuzzy graph is analyzed which is used to find 
the spreading rate of virus. This paper is organized as follows. In Section 2, we give the required definitions. In Section 3, we define the Max-Min intuitionistic fuzzy matrix $M(G)$ of an intuitionistic fuzzy graph. And the extreme energy of $M(G)$ is defined. And we give the explicit expression for the coefficients of the characteristic polynomial of $M(G)$. In Section 4, we illustrate these concepts with real time example. In Section 5, we give the conclusion.

\section{Preliminaries}

\subsection{Intuitionistic Fuzzy Graph}

Definition 1. (see [15]) An intuitionistic fuzzy graph is defined as $G=$ $(V, E, \mu, \gamma)$ where $V$ is the set of vertices and $E$ is the set of edges. $\mu$ is a fuzzy membership function defined on $V \times V$ and $\gamma$ is a fuzzy non - membership function defined on $V \times V$. We denote $\mu\left(v_{i}, v_{j}\right)$ by $\mu_{i j}$ and $\gamma\left(v_{i}, v_{j}\right)$ by $\gamma_{i j}$ such that:

(i) $0 \leq \mu_{i j}+\gamma_{i j} \leq 1$;

(ii) $0 \leq \mu_{i j}, \gamma_{i j}, \pi_{i j} \leq 1$, where $\pi_{i j}=1-\mu_{i j}-\gamma_{i j}$.

Hence $(V \times V, \mu, \gamma)$ is an intuitionistic fuzzy set.

Definition 2. (see [15]) An intuitionistic fuzzy adjacency matrix of an intuitionistic fuzzy graph is defined as the adjacency matrix of the corresponding intuitionistic fuzzy graph. That is for an intuitionistic fuzzy graph $G=$ $(V, E, \mu, \gamma)$, an intuitionistic fuzzy adjacency matrix is defined by $A(I G)=\left[a_{i j}\right]$ where $a_{i j}=\left(\mu_{i j}, \gamma_{i j}\right)$. Note that $\mu_{i j}$ represents the strength of the relationship between $v_{i}$ and $v_{j}$ and $\gamma_{i j}$ represents the strength of the non-relationship between $v_{i}$ and $v_{j}$. The adjacency matrix of an intuitionistic fuzzy graph can be written as two matrices one containing the entries as membership values and the other containing the entries as non-membership values. i.e. $A(I G)=$ $\left(\left(\mu_{i j}\right),\left(\gamma_{i j}\right)\right)$.

\subsection{Energy of an Intuitionistic Fuzzy Graph}

Definition 3. (see [15]) The energy of an intuitionistic fuzzy graph $G=$ $(V, E, \mu, \gamma)$ is defined as $\left(\sum_{\lambda_{i} \in X}\left|\lambda_{i}\right|, \sum_{\delta_{i} \in Y}\left|\delta_{i}\right|\right)$ where $\sum_{\lambda_{i} \in X}\left|\lambda_{i}\right|$ is defined as an energy of the membership matrix denoted by $E\left(\mu_{i j}(G)\right)$ and $\sum_{\delta_{i} \in Y}\left|\delta_{i}\right|$ is defined as an energy of the non - membership matrix denoted by $E\left(\gamma_{i j}(G)\right)$. 
Theorem 4. (see [15]) Let $G$ be an intuitionistic fuzzy directed graph (without loops) with $|V|=n$ and $|E|=m$ and $A(I G)=\left(\left(\mu_{i j}\right),\left(\gamma_{i j}\right)\right)$ be an intuitionistic fuzzy adjacency matrix of $G$ then:

(i)

$$
\sqrt{2 \sum_{1 \leq i<j \leq n} \mu_{i j} \mu_{j i}+n(n-1)|A|^{\frac{2}{n}}} \leq E\left(\mu_{i j}(G)\right) \leq \sqrt{2 n \sum_{1 \leq i<j \leq n} \mu_{i j} \mu_{j i}}
$$

where $|A|$ is the determinant of $A\left(\mu_{i j}\right)$, and

(ii)

$$
\sqrt{2 \sum_{1 \leq i<j \leq n} \gamma_{i j} \gamma_{j i}+n(n-1)|B|^{\frac{2}{n}}} \leq E\left(\gamma_{i j}(G)\right) \leq \sqrt{2 n \sum_{1 \leq i<j \leq n} \gamma_{i j} \gamma_{j i}}
$$

where $|B|$ is the determinant of $A\left(\gamma_{i j}\right)$.

\subsection{Virus Spread in an Intuitionistic Fuzzy Network}

Definition 5. (see [7]) If $\left(E\left(\mu_{i j}(G)\right)\right)>\left(E\left(\gamma_{i j}(G)\right)\right)$ i.e. If the number of visitors are maximum in an intuitionistic fuzzy network then the spreading rate of virus will be maximum. If $\left(E\left(\mu_{i j}(G)\right)\right)>\left(E\left(\gamma_{i j}(G)\right)\right)$ then the infection rate of an intuitionistic fuzzy network is defined as $\beta=\max _{i, j} \mu_{i j}$ and the curing rate of an intuitionistic fuzzy network is defined as $\delta=\min _{i, j} \gamma_{i j}$. The ratio $\tau=$ $\frac{\beta}{\delta}(\delta \neq 0)$ is the effective spreading rate and $\tau_{c}=\frac{1}{\lambda_{\max } A\left(\mu_{i j}\right)}$ where $\lambda_{\max } A\left(\mu_{i j}\right)$ is the largest eigen value of the adjacency matrix $A\left(\mu_{i j}\right)$ of an intuitionistic fuzzy network.

Definition 6. (see [7]) If $\left(E\left(\mu_{i j}(G)\right)\right)<\left(E\left(\gamma_{i j}(G)\right)\right)$ i.e. If the number of visitors are minimum in an intuitionistic fuzzy network then the spreading rate of virus will be minimum. If $\left(E\left(\mu_{i j}(G)\right)\right)<\left(E\left(\gamma_{i j}(G)\right)\right)$ then the infection rate of an intuitionistic fuzzy network is defined as $\beta=\min _{i, j} \mu_{i j}$ and the curing rate of an intuitionistic fuzzy network is defined as $\delta=\max _{i, j} \gamma_{i j}$. The ratio $\tau=\frac{\beta}{\delta}(\delta \neq 0)$ is the effective spreading rate and $\tau_{c}=\frac{1}{\lambda_{\max } A\left(\gamma_{i j}\right)}$ where $\lambda_{\max } A\left(\gamma_{i j}\right)$ is the largest eigen value of the adjacency matrix $A\left(\gamma_{i j}\right)$ of an intuitionistic fuzzy network. 


\section{Max-Min Intuitionistic Fuzzy Matrix of an Intuitionistic Fuzzy Graph}

In this section we define the Max-Min intuitionistic fuzzy matrix $M(G)$ of an intuitionistic fuzzy graph. And the extreme energy of $M(G)$ is defined. And we give the explicit expression for the coefficients of the characteristic polynomial of $M(G)$.

Definition 7. Let $G=(V, E, \mu, \gamma)$ be an intuitionistic fuzzy graph. $\forall$ node $i$, define $\alpha_{j}=\max _{i} \mu_{i j}$ and $\sigma_{j}=\min _{i} \gamma_{i j}$.

Definition 8. Let $G=(V, E, \mu, \gamma)$ be an intuitionistic fuzzy graph. The Max-Min intuitionistic fuzzy matrix of an intuitionistic fuzzy graph is defined as $M(G)=\left(\left(r_{i j}\right),\left(s_{i j}\right)\right)$, where

$$
r_{i j}= \begin{cases}\max \left(\alpha_{i}, \alpha_{j}\right), & \text { if } \mu_{i j} \neq 0 \\ 0, & \text { otherwise }\end{cases}
$$

and

$$
s_{i j}= \begin{cases}\min \left(\sigma_{i}, \sigma_{j}\right), & \text { if } \gamma_{i j} \neq 0, \\ 0, & \text { otherwise. }\end{cases}
$$

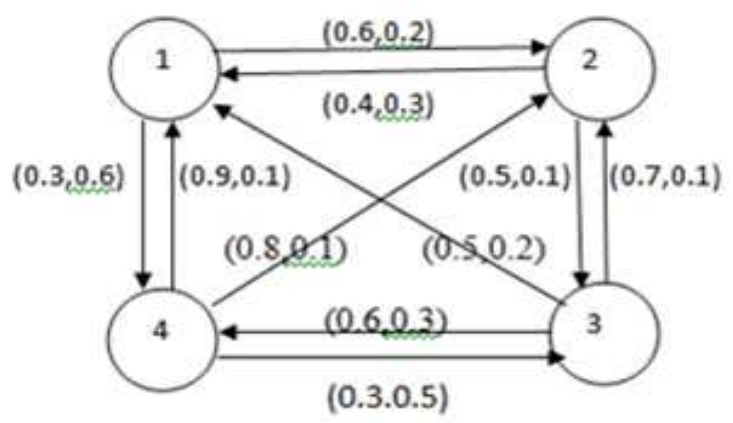

Figure 1: G1,An intuitionistic fuzzy graph

Example 9. For the graph in Figure 1, the Max-Min intuitionistic fuzzy 
matrix is

$$
M(G)=\left(\begin{array}{cccc}
0 & (0.9,0.1) & 0 & (0.9,0.1) \\
(0.9,0.1) & 0 & (0.8,0.1) & 0 \\
(0.9,0.1) & (0.8,0.1) & 0 & (0.6,0.1) \\
(0.9,0.1) & (0.8,0.1) & (0.6,0.1) & 0
\end{array}\right)
$$

where

$$
\left(r_{i j}\right)=\left(\begin{array}{cccc}
0 & 0.9 & 0 & 0.9 \\
0.9 & 0 & 0.8 & 0 \\
0.9 & 0.8 & 0 & 0.6 \\
0.9 & 0.8 & 0.6 & 0
\end{array}\right)
$$

and

$$
\left(s_{i j}\right)=\left(\begin{array}{cccc}
0 & 0.1 & 0 & 0.1 \\
0.1 & 0 & 0.1 & 0 \\
0.1 & 0.1 & 0 & 0.1 \\
0.1 & 0.1 & 0.1 & 0
\end{array}\right)
$$

Let $M(G)=\left(\left(r_{i j}\right),\left(s_{i j}\right)\right)$ be the Max-Min intuitionistic fuzzy matrix of an intuitionistic fuzzy graph $G$. Let $R=\left(r_{i j}\right)$ and $S=\left(s_{i j}\right)$. The characteristic polynomial of $R$ and $S$ is an equation of order $n$ if $G$ has $n$ nodes. For our further discussion we confine our self the polynomial of order 4 . The Characteristic polynomial of $R$ of order 4 is $c_{0} \theta^{4}-c_{1} \theta^{3}+c_{2} \theta^{2}-c_{3} \theta+c_{4}=0$ where $c_{0}=1, c_{1}=$ $\operatorname{tr}(R), c_{2}=\frac{1}{2}\left((\operatorname{tr} R)^{2}-\operatorname{tr}\left(R^{2}\right)\right), c_{3}=\frac{1}{6}\left((\operatorname{tr} R)^{3}-3 \operatorname{tr}\left(R^{2}\right)(\operatorname{tr} R)+2 \operatorname{tr}\left(R^{3}\right)\right)$ and $c_{4}=\operatorname{det}(R)$. We now give the explicit expression for the coefficients of $c_{2}$ and $c_{3}$.

Definition 10. Mutually adjacent. Let $G$ be the given intuitionistic fuzzy graph. Two vertices $v_{i}$ and $v_{j}$ are said to be mutually adjacent if there is an edge from $v_{i}$ to $v_{j}$ and there is an edge from $v_{j}$ to $v_{i}$.

Definition 11. Cyclic. Let $G$ be the given intuitionistic fuzzy graph. Three vertices $v_{i}, v_{j}$ and $v_{k}$ are cyclic if there is an edge from $v_{i}$ to $v_{j}, v_{j}$ to $v_{k}$ and $v_{k}$ to $v_{i}$.

Lemma 12. In the characteristic polynomial of $R, c_{2}=-\sum_{1 \leq i<j \leq n}\left(r_{i j}\right)^{2}$ if $v_{i}$ and $v_{j}$ are mutually adjacent.

Proof. In general we have, $c_{2}=\frac{1}{2}\left((\operatorname{tr} R)^{2}-\operatorname{tr}\left(R^{2}\right)\right)$ Note that if $v_{i}$ and 


$$
\begin{gathered}
=\sum_{1 \leq i<j \leq n}\left|\begin{array}{cc}
0 & r_{i j} \\
r_{j i} & 0
\end{array}\right| \\
=-\left[r_{12} r_{21}+r_{13} r_{31}+r_{14} r_{41}+r_{23} r_{32}+r_{24} r_{42}+r_{34} r_{43}\right] .
\end{gathered}
$$

$v_{j}$ are mutually adjacent, then $r_{i j}=r_{j i}$ otherwise any one of $r_{i j}$ or $r_{j i}$ will be zero. Therefore (1) becomes $c_{2}=-\sum_{1 \leq i<j \leq n}\left(r_{i j}\right)^{2}$ if $v_{i}$ and $v_{j}$ are mutually adjacent.

Lemma 13. In the characteristic polynomial of $R, c_{3}=\sum r_{i j} r_{j k} r_{k i}$ where the summation is taken over all $i, j$ and $k$ such that $v_{i}, v_{j}$ and $v_{k}$ are cyclic in $G$.

Proof. In general we have, $c_{3}=\frac{1}{6}\left((\operatorname{tr} R)^{3}-3 \operatorname{tr}\left(R^{2}\right)(\operatorname{tr} R)+2 \operatorname{tr}\left(R^{3}\right)\right)$

$$
\begin{gathered}
=\sum_{1 \leq i<j<k \leq n}\left|\begin{array}{ccc}
r_{i i} & r_{i j} & r_{i k} \\
r_{j i} & r_{j j} & r_{j k} \\
r_{k i} & r_{k j} & r_{k k}
\end{array}\right| \\
=r_{12} r_{23} r_{31}+r_{12} r_{24} r_{41}+r_{13} r_{34} r_{41}+r_{13} r_{32} r_{21}+r_{14} r_{43} r_{31} \\
+r_{14} r_{42} r_{21}+r_{23} r_{34} r_{42}+r_{24} r_{43} r_{32} .
\end{gathered}
$$

Note that if $v_{i}, v_{j}$ and $v_{k}$ are cyclic then $c_{3}=\sum r_{i j} r_{j k} r_{k i}$ otherwise any one of $r_{i j}$ or $r_{j k}$ or $r_{k i}$ will be zero. Therefore (2) becomes $c_{3}=\sum r_{i j} r_{j k} r_{k i}$ where the summation is taken over all $i, j$ and $k$ such that $v_{i}, v_{j}$ and $v_{k}$ are cyclic in $G$.

Theorem 14. (see [5]) If $\theta_{1}, \theta_{2}, \ldots, \theta_{n}$ are the eigen values of $R$, then $\sum_{i=1}^{n} \theta_{i}^{2}=-2 c_{2}$.

Proof. We know that

$$
\begin{gathered}
\left(\sum_{i=1}^{n} \theta_{i}\right)^{2}=\sum_{i=1}^{n} \theta_{i}^{2}+2 \sum_{1 \leq i<j \leq n} \theta_{i} \theta_{j} \\
0=\sum_{i=1}^{n} \theta_{i}^{2}+2 \sum_{1 \leq i<j \leq n} \theta_{i} \theta_{j}
\end{gathered}
$$


By comparing the coefficients of $\theta^{n-2}$ in the characteristic polynomial

$$
\prod_{i=1}^{n}\left(\theta-\theta_{i}\right)=|A-\theta I| .
$$

We get

$$
\sum_{1 \leq i<j \leq n} \theta_{i} \theta_{j}=-\sum_{1 \leq i<j \leq n} r_{i j} r_{j i}
$$

Substituting (4) in (3), we get

$$
\begin{gathered}
\sum_{i=1}^{n} \theta_{i}^{2}=2 \sum_{1 \leq i<j \leq n} r_{i j} r_{j i} \\
=2\left[r_{12} r_{21}+r_{13} r_{31}+r_{14} r_{41}+r_{23} r_{32}+r_{24} r_{42}+r_{34} r_{43}\right]
\end{gathered}
$$

Note that if $v_{i}$ and $v_{j}$ are mutually adjacent, then $r_{i j}=r_{j i}$ otherwise any one of $r_{i j}$ or $r_{j i}$ will be zero. Therefore (5) becomes $\sum_{i=1}^{n} \theta_{i}^{2}=2 \sum_{1 \leq i<j \leq n}\left(r_{i j}\right)^{2}$ if $v_{i}$ and $v_{j}$ are mutually adjacent. i.e. $\sum_{i=1}^{n} \theta_{i}^{2}=-2 c_{2}$.

From the above discussion all the coefficients of the characteristic polynomial of matrix $R$ can be calculated. Now let us consider the matrix $S$ whose characteristic polynomial is $d_{0} \lambda^{4}-d_{1} \lambda^{3}+d_{2} \lambda^{2}-d_{3} \lambda+d_{4}=0$ here also $d_{0}=1, d_{1}=\operatorname{tr}(S), d_{2}=-\sum_{1 \leq i<j \leq n}\left(s_{i j}\right)^{2}$ if $v_{i}$ and $v_{j}$ are mutually adjacent, $d_{3}=\sum s_{i j} s_{j k} s_{k i}$, where the summation is taken over all $i, j$ and $k$ such that $v_{i}, v_{j}$ and $v_{k}$ are cyclic in $G$ and $d_{4}=\operatorname{det}(S)$. If $\lambda_{1}, \lambda_{2}, \ldots, \lambda_{n}$ are the eigen values of $S$, then $\sum_{i=1}^{n} \lambda_{i}^{2}=-2 d_{2}$.

Definition 15. Extreme energy of an intuitionistic fuzzy graph. The extreme energy of an intuitionistic fuzzy graph $G=(V, E, \mu, \gamma)$ is defined by $\left(E_{1}, E_{2}\right)$ where $E_{1}$ is the energy of the Max degree matrix $R$ and $E_{2}$ is the energy of the Min degree matrix $S$.

Example 16. The Characteristic polynomial of $R$ is $\theta^{4}-(2.62) \theta^{2}-$ (2.166) $\theta-0.486$.

Here $c_{0}=1, c_{1}=\operatorname{tr}(R)=0, c_{4}=\operatorname{det}(R)=-0.486, c_{2}=-\sum_{1 \leq i<j \leq n}\left(r_{i j}\right)^{2}$ if $v_{i}$ and $v_{j}$ are mutually adjacent. i.e.

$$
c_{2}=-\left[(0.9)^{2}+0+(0.9)^{2}+(0.8)^{2}+0+(0.6)^{2}\right]=-2.62
$$

and $c_{3}=\sum r_{i j} r_{j k} r_{k i}$ where the summation is taken over all $i, j$ and $k$ such that $v_{i}, v_{j}$ and $v_{k}$ are cyclic in $G$. i.e.

$c_{3}=(0.9)(0.8)(0.9)+(0.9)(0.6)(0.9)+(0.9)(0.8)(0.9)+(0.8)(0.6)(0.8)=2.166$. 
Also

$$
\begin{array}{r}
\sum_{i=1}^{n} \theta_{i}^{2}=\left[(1.9622)^{2}+0+(-0.9071)^{2}+(-0.4551)^{2}+0+(-0.6000)^{2}\right] \\
=5.24=-2 c_{2} .
\end{array}
$$

The Characteristic polynomial of $S$ is $\lambda^{4}-(0.04) \lambda^{2}-(0.004) \lambda-0.0001$.

Here $d_{0}=1, d_{1}=\operatorname{tr}(S)=0, d_{4}=\operatorname{det}(S)=-0.0001, d_{2}=-\sum_{1 \leq i<j \leq n}\left(s_{i j}\right)^{2}$ if $v_{i}$ and $v_{j}$ are mutually adjacent. i.e.

$$
d_{2}=-\left[(0.1)^{2}+0+(0.1)^{2}+(0.1)^{2}+0+(0.1)^{2}\right]=-0.04
$$

and $d_{3}=\sum s_{i j} s_{j k} s_{k i}$ where the summation is taken over all $i, j$ and $k$ such that $v_{i}, v_{j}$ and $v_{k}$ are cyclic in $G$. i.e.

$d_{3}=(0.1)(0.1)(0.1)+(0.1)(0.1)(0.1)+(0.1)(0.1)(0.1)+(0.1)(0.1)(0.1)=0.004$.

Also

$$
\begin{array}{r}
\sum_{i=1}^{n} \lambda_{i}^{2}=\left[(0.2414)^{2}+0+(-0.1000)^{2}+(-0.0414)^{2}+0+(-0.1000)^{2}\right] \\
=0.08=-2 d_{2} .
\end{array}
$$

Extreme energy of an intuitionistic fuzzy graph is $\left(E_{1}, E_{2}\right)=(3.9243,0.4828)$.

\section{Numerical Examples}

In this section we give the extreme energy of an intuitionistic fuzzy graph through real time example. We have taken the website http://www.pantechso lutions.net/. This website is modeled as an intuitionistic fuzzy graph by considering the navigation of the customer. An intuitionistic fuzzy graph of this site for four different time periods is taken. For each of these periods the Max-Min intuitionistic fuzzy matrix is constructed and the extreme energy is calculated. These results are represented in the table .

Example 17. In the website http://www.pantechsolutions.net/ we consider four links 1.microcontroller-boards, 2./log-in html, 3./ and 4. project kits for the period July 16, 2013 to August 15, 2013.

For an intuitionistic fuzzy graph in Figure 2,

Extreme energy $=(0.8745,0.9479)$ but by consider the intuitionistic fuzzy adjacency matrix $A\left(G_{2}\right)$ of this graph, the energy $=(0.4,2.1078)$ (see [15]). 


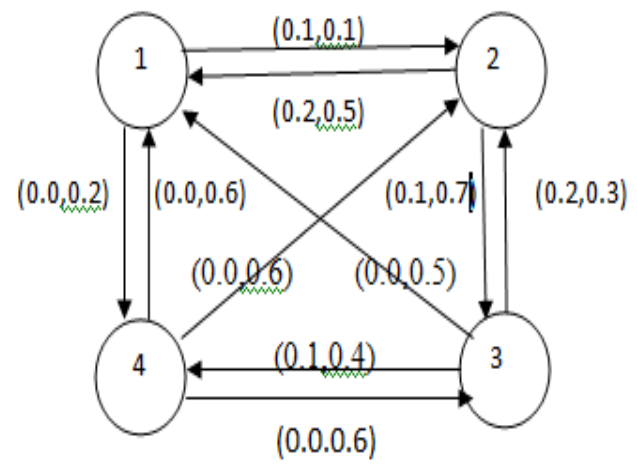

Figure 2: G2,An intuitionistic fuzzy graph

Example 18. In the same website (mentioned above in example 17), we consider the same four links for the period August 16, 2013 to September 15, 2013.

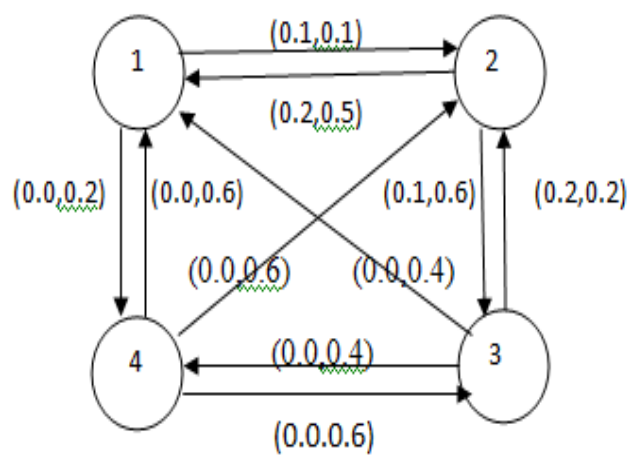

Figure 3: G3,An intuitionistic fuzzy graph

For an intuitionistic fuzzy graph in Figure 3,

Extreme energy $=(0.8745,0.8902)$ but by consider the intuitionistic fuzzy adjacency matrix $A\left(G_{3}\right)$ of this graph, the energy $=(0.4,1.9096)$ (see [15]).

Example 19. In the same website (mentioned above in example 17), we consider the same four links for the period September 16, 2013 to October 15, 2013.

For an intuitionistic fuzzy graph in Figure 4,

Extreme energy $=(0.7427,0.9479)$ but by consider the intuitionistic fuzzy 


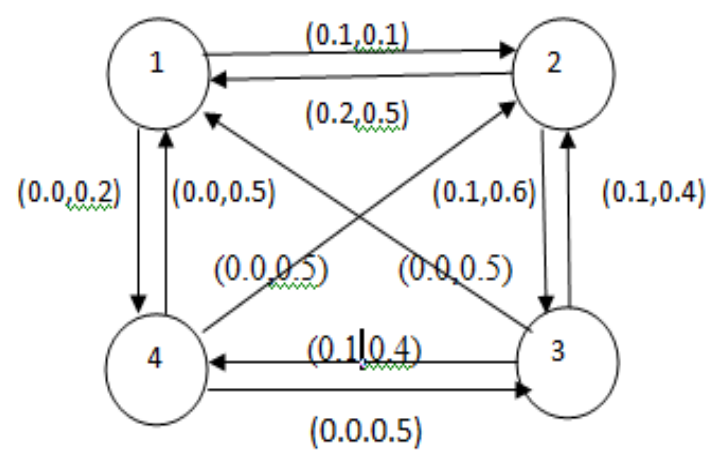

Figure 4: G4,An intuitionistic fuzzy graph

adjacency matrix $A\left(G_{4}\right)$ of this graph, the energy $=(0.3464,1.913)$ (see [15]).

Example 20. In the same website (mentioned above in example 17), we consider the same four links for the period of October 16, 2013 to November 15, 2013.

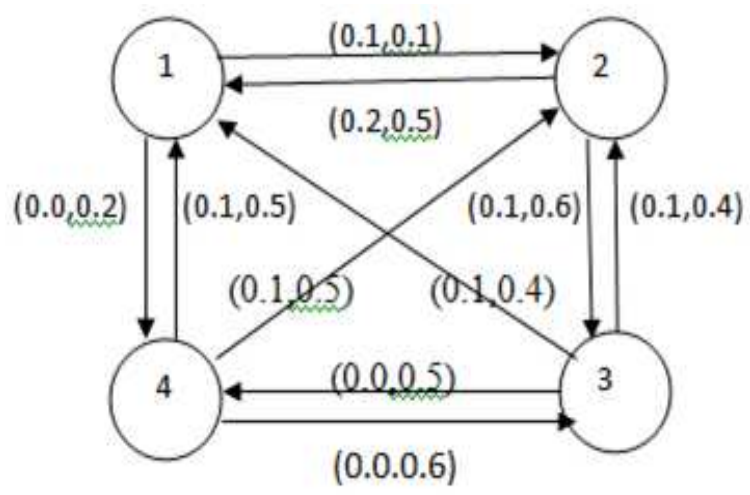

Figure 5: G5,An intuitionistic fuzzy graph

For an intuitionistic fuzzy graph in Figure 5,

Extreme energy $=(0.7427,0.8902)$ but by consider the intuitionistic fuzzy adjacency matrix $A\left(G_{5}\right)$ of this graph, the energy $=(0.3758,1.988)$ (see $\left.[15]\right)$.

The following table represents the comparison of energy of $A(G)$ and the Extreme energy of $M(G)$.

From the above table we can observe that the energy of $\mu$ of $A(G)$ for all the time periods is < then the Extreme energy of $R$ in $M(G)$. Similarly the 


\begin{tabular}{|c|c|c|}
\hline period & Energy of $A(G)$ & Extreme energy of $M(G)$ \\
\hline Jul-Aug & $(0.4,2.1078)$ & $(0.8745,0.9479)$ \\
\hline Aug-Sep & $(0.4,1.9096)$ & $(0.8745,0.8902)$ \\
\hline Sep-Oct & $(0.3464,1.913)$ & $(0.7427,0.9479)$ \\
\hline Oct-Nov & $(0.3758,1.988)$ & $(0.7427,0.8902)$ \\
\hline
\end{tabular}

energy of $\gamma$ of $A(G)>$ then the Extreme energy of $S$ in $M(G)$.

\section{Conclusion}

In this paper we introduced the Max-Min intuitionistic fuzzy matrix $M(G)$ of an intuitionistic fuzzy graph $G$. And the extreme energy of $M(G)$ is defined. And also we give the explicit expression for the coefficients of the characteristic polynomial of $M(G)$. These concepts are illustrated through real time example. These results are compared with the energy of an intuitionistic fuzzy graph.

\section{Acknowledgments}

The author thank the management for their support towards the completion of this work.

\section{References}

[1] N. Anjali, Sunil Mathew, Energy of a fuzzy graph, Annals of Fuzzy Mathematics and Informatics, (2013).

[2] K. Atanassov, Intuitionistic Fuzzy sets, Theory and Applications, SpringerVerlag, Heidelberg, (1999).

[3] N.T.J. Bailey, The Mathematical Theory of Infectious Diseases and its applications, 2nd ed. London, U.K, Charlin Griffin, (1975).

[4] R.A. Brualdi, Energy of a graph, Notes to AIM Workshop on spectra of families of atrices described by graphs, digraphs and sign patterns, (2006).

[5] Chandrashekar Adiga and M. Smitha, On Maximum degree energy of a graph, Int. J. Contemp. Math. Sci., 4(5-8)(2009), 385-396. 
[6] D.J. Daley, and J. Gani, Epidemic Modelling: An Introduction, Cambridge University Press, Cambridge, (1999).

[7] G. Deepa, B. Praba, V.M. Chandrasekaran, Virus Spread in an Intuitionistic Fuzzy Network, International Journal of Applied Engineering Research, Vol.9, No.16, (2014), 5507-5515.

[8] I. Gutman, The energy of a graph, Ber. Math. Statist. Sekt. Forschungszentram Graz. 103, (1978), 122.

[9] I. Gutman, A. Betten, A. Kohner, R. Laue, and A.Wassermann,eds., The energy of a graph: old and new results, Algebraic Combinatorics and Applications,Springer, Berlin, (2001), 196-211.

[10] A. Kauffman, Introduction a la Theorie des sous ensembles Flous, Masson et cie., Vol.1.(1973).

[11] J.O. Kephart, and R S. White, Directed-Graph Epidemiological models of Computer Viruses, Proc. IEEE Comput. Soc. Symp. Research in Security and Privacy, (1991), 343-359.

[12] H. Liu, M. Lu and F. Tian, Some upper bounds for the energy of graphs, J. Math. Chem. 42, (2007), 377-386.

[13] R. Pastor - Satorras and A. Vespignani, Epidemic Spreading in Scale free networks, Phys. Rev. Lett, Vol. 86, no. 14, (2001), 3200-3203.

[14] B. Praba, and R. Sujatha, Application of Fuzzy Markov model for webtesting, Ganita Sandesh, vol. 21(2), (2007), 111-120.

[15] B. Praba, V.M. Chandrasekaran, G. Deepa, Energy of an Intuitionistic Fuzzy Graph, Italian journal of pure and applied mathematics, N.32, (2014), 431-444.

[16] A. Rosenfeld, Fuzzy graphs, in L. A. Zadeh, K. cS. Fu, K. Tanaka and M. Shimura, eds, Fuzzy sets and their applications to cognitive and decision process, Academic press, New York, (1975), 75-95.

[17] P. Van Mieghem, Member, IEEE, Jasmina Omic and Robert Kooij, Virus Spread in Networks, IEEE/ACM Transaction on Networking, Vol. 17, No. 1, (2009). 
